\title{
Exploration of serum cardiac troponin I as a biomarker of cardiomyopathy in southern sea otters (Enhydra lutris nereis)
}

\author{
Megan E. Moriarty DVM, MPVM, PhD \\ Melissa A. Miller DVM, PhD \\ Michael J. Murray DVM \\ Pádraig J. Duignan DVM, PhD \\ Catherine T. Gunther-Harrington DVM \\ Cara L. Field DVM, PhD \\ Lance M. Adams DVM \\ Todd L. Schmitt DVM \\ Christine K. Johnson VMD, MPVM, PhD
}

Received August 21, 2020

Accepted October 6, 2020.

From the Karen C. Drayer Wildlife Health Center and EpiCenter for Disease Dynamics, One Health Institute (Moriarty, Johnson), and Department of Medicine \& Epidemiology (Gunther-Harrington), School of Veterinary Medicine, University of California-Davis, Davis, CA 95616; Marine Wildlife Veterinary Care and Research Center, California Department of Fish and Wildlife, Santa Cruz, CA 95060 (Miller); Monterey Bay Aquarium, Monterey, CA 93940 (Murray); The Marine Mammal Center, Sausalito, CA 94965 (Duignan, Field); Aquarium of the Pacific, Long Beach, CA 90802 (Adams); and SeaWorld Animal Health and Rescue Center, San Diego, CA 92109 (Schmitt).

Address correspondence to Dr. Moriarty (memoriarty @ucdavis.edu).

\begin{abstract}
OBJECTIVE
To compare serum cardiac troponin I ( $c T n l)$ concentrations between sea otters with and without cardiomyopathy and describe 2 cases of cardiomyopathy with different etiologies.
\end{abstract}

\section{ANIMALS}

25 free-ranging southern sea otters (Enhydra lutris nereis) with $(\mathrm{n}=14$; cases) and without (II; controls) cardiomyopathy and 17 healthy managed southern sea otters from aquariums or rehabilitation centers (controls).

\section{PROCEDURES}

Serum cTnl concentration was measured in live sea otters. Histopathologic and gross necropsy findings were used to classify cardiomyopathy status in free-ranging otters; physical examination and echocardiography were used to assess health status of managed otters. Two otters received extensive medical evaluations under managed care, including diagnostic imaging, serial cTnl concentration measurement, and necropsy.

\section{RESULTS}

A significant difference in cTnl concentrations was observed between cases and both control groups, with median values of $0.279 \mathrm{ng} / \mathrm{mL}$ for cases and $<0.006 \mathrm{ng} / \mathrm{mL}$ for free-ranging and managed controls. A cutoff value of $\geq$ $0.037 \mathrm{ng} / \mathrm{mL}$ yielded respective sensitivity and specificity estimates for detection of cardiomyopathy of $64.3 \%$ and $90.9 \%$ for free-ranging cases versus free-ranging controls and $64.3 \%$ and $94.1 \%$ for free-ranging cases versus managed controls.

\section{CONCLUSIONS AND CLINICAL RELEVANCE}

Cardiomyopathy is a common cause of sea otter death that has been associated with domoic acid exposure and protozoal infection. Antemortem diagnostic tests are needed to identify cardiac damage. Results suggested that serum cTnl concentration has promise as a biomarker for detection of cardiomyopathy in sea otters. Serial cTnl concentration measurements and diagnostic imaging are recommended to improve heart disease diagnosis in managed care settings. (Am J Vet Res 2021;82:529-537)
$S^{\text {o }}$ uthern sea otters (Enbydra lutris nereis) are a federally listed threatened species that is intensively managed to help ensure population recovery. A study ${ }^{1}$ found that cardiomyopathy was a primary or contributing cause of death in 41\% (229/552) of free-ranging otters that underwent necropsy from 1998 to 2012 . This prevalent condition is often identified during postmortem examination, and antemortem diagnosis remains challenging, owing in part to a lack of overt clinical signs in the early to mid stages of cardiomyopathy and a scarcity of analytic tools. The southern sea otter research, rescue, and rehabilitation community was in a unique position

$\begin{array}{ll}\text { ABBREVIATIONS } \\ \text { AUC } & \text { Area under the curve } \\ \text { cTnl } & \text { Cardiac troponin I } \\ \text { DA } & \text { Domoic acid } \\ \text { ROC } & \text { Receiver operating characteristic } \\ \text { VHS } & \text { Vertebral heart scale }\end{array}$

to bridge this knowledge gap by combining information from comprehensive necropsies and clinical case management of otters at aquariums and rehabilitation centers.

Cardiomyopathy in sea otters is a multifactorial condition, with protozoal infection and DA toxin exposure identified as risk factors. ${ }^{1-3}$ The protozoan parasites Toxoplasma gondii and Sarcocystis neurona cause disease in the CNS but are also associated with cardiac abnormalities. ${ }^{1,2,4,5}$ Necropsy data show that otters infected with $S$ neurona and $T$ gondii, as determined by serologic and histologic evaluation, have higher odds of cardiomyopathy than do uninfected otters. ${ }^{1}$ Domoic acid is a neurotoxin produced by harmful algal blooms and an important cause of death in marine mammals along the Pacific Coast of North America. ${ }^{6}$ This toxin causes neurologic and gastrointestinal disease, but cardiovascular effects have also been recognized in southern sea otters, ${ }^{1,2,7}$ 
California sea lions (Zalopbus californianus), ${ }^{8,9}$ and humans. ${ }^{10,11}$ Postmortem examinations have revealed that sea otters with DA-associated CNS abnormalities often have cardiovascular lesions, and these cases may represent acute, subacute, or chronic DA exposure. ${ }^{7}$ In these animals, DA-associated cardiomyopathy is progressive and represents a spectrum of disease, with dilated cardiomyopathy on the far end of the lesion continuum. ${ }^{7}$ Domoic acid is rapidly cleared after ingestion, and current diagnostic methods cannot assess repeated or chronic exposure. ${ }^{12,13}$ Furthermore, no accurate antemortem clinical indicator has been identified for detection of acute DA toxicosis or its more chronic effects such as cardiomyopathy. This has impeded the ability to provide optimal medical care to sea otters in various clinical settings. Reliable biomarkers are needed to confirm a diagnosis, provide prognostic information, and identify cardiac damage before it progresses to dilated cardiomyopathy.

Cardiac troponins are blood-based biomarkers of heart disease that are widely used in human and veterinary medicine. Cardiac troponin $I$ is part of a regulatory protein complex in the contractile fibers of cardiomyocytes that controls the calcium-mediated interaction between actin and myosin. ${ }^{14}$ It is a highly conserved biomarker of myocardial injury in mammals detectable by an immunoassay with high sensitivity for myocardial damage. ${ }^{15-18}$ Cardiac troponin I has many qualities of an ideal biomarker, such as cardiac specificity, negligible presence in healthy individuals, rapid release, and continued presence in the circulation for days after damage. ${ }^{15}$ High serum cTnI concentrations are correlated with greater disease severity and a worse prognosis in dogs. ${ }^{16,19}$ Finally, several cTnI assays are commercially available and can enable rapid measurement of cTnI concentration in serum or plasma. ${ }^{20,21}$

In previous research, ${ }^{22}$ we performed transthoracic echocardiography and measured serum cTnI concentrations in 15 anesthetized healthy female southern sea otters. That research established a foundation for our next study, the aim of which was to assess the clinical utility of a widely conserved biomarker for myocardial damage in sea otters. Our objectives for the study reported here were to compare cTnI concentration in antemortem serum samples from southern sea otters with cardiomyopathy (cases) and 2 groups without cardiac disease (controls) and to describe cardiomyopathy of different etiologies in 2 sea otters to demonstrate the value of serial cTnI testing in managed care settings where periodic physical examination and diagnostic testing are feasible.

\section{Materials and Methods}

\section{Ethics statement}

This study was conducted with approval by the Institutional Animal Care and Use Committee of the University of California-Davis and Monterey Bay
Aquarium. Animal housing facilities complied with USDA regulations governing welfare of marine mammals and met Association of Zoos and Aquariums accreditation standards.

\section{Animals}

Free-ranging southern sea otters that had become stranded and then died or were euthanized and that had cardiomyopathy as a primary or contributing cause of death were selected as cases. Unless otherwise stated, all necropsies, including gross and histologic evaluation of major organs and tissues, ${ }^{1}$ were performed by a veterinary pathologist at the California Department of Fish and Wildlife. All cases had microscopically confirmed moderate to severe cardiac disease; sea otters with mild cardiomyopathy were excluded. The pathologist graded lesion severity and chronicity as well as the likelihood of DA involvement in cardiac disease, including an assessment of DA-associated brain abnormalities. Determination of DA toxicosis was based on a postmortem case definition for sea otters that included standardized protocols for gross necropsy, microscopic examination, and diagnostic testing of biological samples. ${ }^{1,7}$ When available, antemortem data were also used. In live sea otters, clinical signs and biochemical test results for urine and feces can help assess DA exposure, ${ }^{7}$ although clinical signs are often nonspecific and diagnostic tests lack sensitivity for low-level or historical exposure. ${ }^{23}$ Case evaluation also included a cardiac inflammation severity grade (mild, moderate, or marked), predominant inflammatory cell type (lymphoplasmacytic or pleocellular), and whether protozoal cysts, schizonts, or zoites were present in heart, brain, or skeletal muscle tissue.

Two other groups of sea otters were used as controls for comparisons of serum cTnI concentrations with those of the cases. The first control group included free-ranging sea otters that had become stranded and then died or were euthanized and in which postmortem examination revealed no cardiac disease, DA toxicosis, or protozoal infection. Animals with disseminated infection or disease were excluded as controls because noncardiac systemic disease can increase serum cTnI concentration. ${ }^{24}$ The second control group included healthy managed sea otters from aquariums and rehabilitation centers in California, including the Monterey Bay Aquarium, The Marine Mammal Center, Aquarium of the Pacific, and SeaWorld. Echocardiography was performed during wellness examinations for exhibit otters (except for 2 individuals) and prerelease examinations for rehabilitation otters, and no evidence of cardiac disease was identified. ${ }^{22}$ All otters in rehabilitation were healthy at the time of sample collection, and those with previous neurologic, renal, or protozoal disease were excluded.

\section{Sample collection and diagnostic testing}

Venous blood samples were collected from live sea otters and centrifuged; harvested serum was 
stored at $-80^{\circ} \mathrm{C}$ until shipment to the Gastrointestinal Laboratory at Texas A\&M University for analysis. Sample collection from free-ranging cases and controls occurred within 1 month before death, typically at the time of stranding, at admission to a rehabilitation program, or just prior to euthanasia. Sample collection from managed controls occurred during wellness examinations for exhibit otters and prerelease examinations for rehabilitation otters. Serum cTnI concentration was measured at the laboratory by use of a highly sensitive, commercially available immunoassay, a as previously described. ${ }^{22}$ Hemolyzed or lipemic samples were excluded because of the potential for false-negative or false-positive results. ${ }^{25} \mathrm{Al}$ though this assay was developed for use with human serum, it is effective at detecting circulating cTnI in canine and feline sera ${ }^{20}$ and is cross-reactive over several animal species. ${ }^{17,20}$ This assay allows for earlier and faster detection of myocardial injury than do conventional assays, with a minimum detectable serum c'TnI concentration of $0.006 \mathrm{ng} / \mathrm{mL} .^{20}$

\section{Statistical analysis}

Descriptive statistics were calculated. Serum cTnI concentrations were compared between cases and controls with the nonparametric 2-sample MannWhitney $U$ test. $^{\text {b }}$ Differences were considered significant at $P$ values $\leq 0.05$. Test performance (sensitivity and specificity for detection of cardiomyopathy) at different cutoff values for cTnI concentration was evaluated by means of ROC curve analysis. ${ }^{c}$ The reference standard test used to assess cardiac disease status was gross and histologic evaluation of cardiac tissue by veterinary pathologists. The ROC curves were used to compare the overall ability of serum cTnI concentration to detect cardiomyopathy over the range of possible cutoff values. ${ }^{\mathrm{c}}$ The AUC for ROC curves is a summary statistic of diagnostic accuracy that helps evaluate a test's ability to discriminate the true state of the patient. ${ }^{26}$ For example, a noninformative test with no discrimination has an AUC of 0.50 , meaning that a randomly selected otter with cardiomyopathy would have a higher cTnI concentration than a randomly selected otter without cardiac disease only $50 \%$ of the time. In contrast, a perfect test has an AUC of 1.0, which would signify complete separation of cTnI values for otters with and without cardiomyopathy.

\section{Results}

\section{Animals}

Overall, 14 free-ranging southern sea otters with cardiomyopathy (10 males and 4 females; 8 adults, 4 aged adults, and 2 subadults; cases), 11 free-ranging otters without cardiomyopathy $(5$ males and 6 females; 7 adults and 4 subadults; controls), and 17 healthy managed otters ( 1 male and 16 females; 10 adults, 2 aged adults, and 5 juveniles or subadults; 11 from exhibits and 6 in rehabilitation; controls) were included in the study.
All free-ranging cases had cardiomyopathy and DA toxicosis as a primary or contributing cause of death. Among this group, the primary cause of death was cardiomyopathy ( $\mathrm{n}=5)$, DA toxicosis (4), white shark (Carcharodon carcharias) bite (2), sarcocystosis (1), fight trauma (1), and pyelonephritis (1). Systemic protozoal disease was a primary or contributing cause of death in 4 cases ( $S$ neurona $[\mathrm{n}=2$ ] or $T$ gondii [2]). Free-ranging controls died of sudden trauma (white shark bite [ $\mathrm{n}=4$ ], boat strike [1], and entanglement [1]), physiologic conditions associated with age and sex (end-lactation syndrome [2] and mating trauma [1]), and acanthocephalan peritonitis (2).

\section{Serum cTnl concentrations}

Mean and median (range) serum c'TnI concentrations were 0.044 and $0.279 \mathrm{ng} / \mathrm{mL}(<0.006$ to 2.582 $\mathrm{ng} / \mathrm{mL}$ ) for free-ranging cases, 0.014 and $<0.006 \mathrm{ng} /$ $\mathrm{mL}(<0.006$ to $0.061 \mathrm{ng} / \mathrm{mL}$ ) for free-ranging controls, and 0.005 and $<0.006 \mathrm{ng} / \mathrm{mL}(<0.006$ to 0.038 $\mathrm{ng} / \mathrm{mL}$ ) for managed controls. The interval between sample collection and death ranged from 0 to 2 days for cases and 0 to 21 days for free-ranging controls.

Although dot plots revealed overlap between cases and controls in serum cTnI concentrations (Figure I), cTnI values differed significantly between freeranging cases and free-ranging controls $(P=0.01)$ and between free-ranging cases and managed controls $(P$ $<0.001$ ). For ROC curve analyses, the AUC for distinguishing between otters with and without cardiomyopathy was 0.80 (95\% CI, 0.62 to 0.97 ) for freeranging cases versus free-ranging controls and 0.87 (95\% CI, 0.74 to 1.00 ) for free-ranging cases versus managed controls, demonstrating good to excellent discrimination (Figure 2). A serum cTnI concentration cutoff value of $\geq 0.037 \mathrm{ng} / \mathrm{mL}$ maximized test sensitivity and specificity for detection of cardiomyopathy in both comparisons. For free-ranging cases versus free-ranging controls, use of this cutoff value resulted in $76 \%$ of otters being correctly classified, $64.3 \%$ sensitivity (95\% CI, 35.1\% to $87.2 \%$ ), and $90.9 \%$ specificity (95\% CI, $58.7 \%$ to $99.8 \%$ ). For free-ranging cases versus managed controls, this resulted in $81 \%$ of otters being correctly classified, $64.3 \%$ sensitivity (95\% CI, 35.1\% to $87.2 \%$ ), and $94.1 \%$ specificity (95\% CI, $71.3 \%$ to $99.9 \%$; Table I).

\section{Clinical case study I: cardiomyopathy associated with DA toxicosis}

A 13-year-old female southern sea otter that had become stranded with end-lactation syndrome in May 2016 was taken to the Monterey Bay Aquarium. Cardiac auscultation revealed a grade $4 / 6$ systolic murmur over the mitral valve region and a regular rhythm. Cardiac size was serially measured on thoracic radiographs by use of the VHS, whereby cardiac dimensions were compared with the length of the fifth thoracic vertebrae as described for ferrets. ${ }^{27}$ Thoracic radiography revealed a normal cardiac sil- 
houette (VHS, 8.2 vertebrae) and mild to moderate interstitial pulmonary pattern. In November, the otter delivered a pup that died the next day. Over the next 7 months, thoracic radiography revealed progressive cardiac enlargement (VHS, 9.6 vertebrae), an increasingly globoid cardiac silhouette, and pulmonary venous distension. Electrocardiographic examination revealed progressively reduced $\mathrm{P}$ wave amplitude, an increased incidence of atrial and ventricular ectopy, and occasional first- and second-degree atrioventricular block. Serum c'TnI concentration was initially low but gradually increased until it peaked (0.412 ng/ $\mathrm{mL}$ ) in July 2017, 9 months after stranding (Supplementary Table SI, available at: avmajournals.avma.

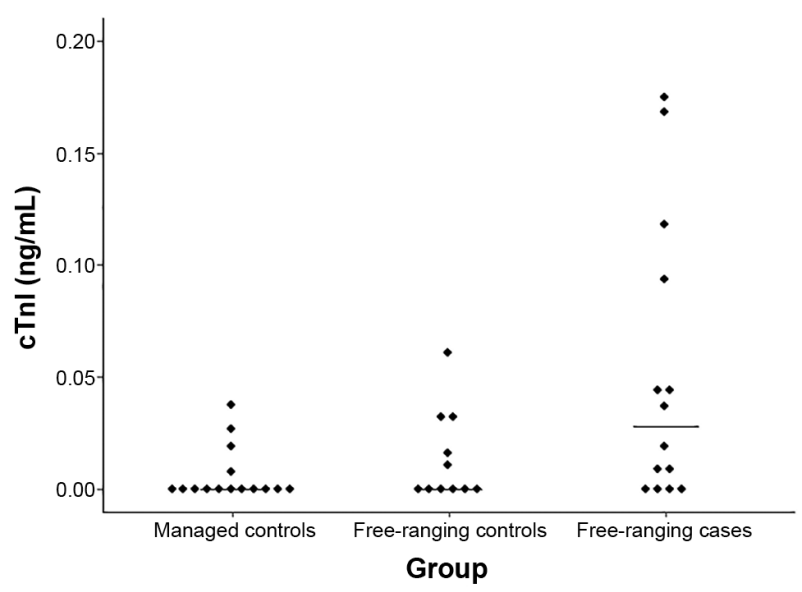

Figure I-Dot plot of serum cTnl concentrations $(\mathrm{ng} / \mathrm{mL})$ in individual free-ranging southern sea otters (Enhydra lutris nereis) with ( $\mathrm{n}=14$; free-ranging cases) and without (II; freeranging controls) cardiomyopathy and healthy managed southern sea otters (17; managed controls). Median concentrations (horizontal lines) differed significantly between free-ranging cases and free-ranging controls $(P=0.0 \mathrm{I})$ and between freeranging cases and managed controls $(P<0.00 \mathrm{I})$. Two outliers with cTnl values of 2.582 and $0.613 \mathrm{ng} / \mathrm{mL}$ (free-ranging cases) have been excluded from this graph. org/doi/suppl/10.2460/ajvr.82.7.529). In August, ECG results were suggestive of atrial ectopy and rare premature ventricular complexes and serum cTnI decreased slightly. In October, the otter's appetite declined and weight loss was noted; thoracic radiography revealed severe progressive cardiomegaly (VHS, 10.5 vertebrae), pulmonary venous distension, and an interstitial to alveolar pulmonary pattern, consistent with congestive heart failure (Supplementary Figure SI, available at: avmajournals.avma.org/doi/ suppl/10.2460/ajvr.82.7.529). The ECG revealed more frequent ventricular ectopy in a bigeminal pattern with very small to absent $P$ waves and a sinoventricular rhythm at a heart rate of 66 beats $/ \mathrm{min}$. The decreasing $\mathrm{P}$ wave amplitude likely represented an atrial myopathy that could have contributed to congestive heart failure. Serum cTnI concentration decreased to $0.054 \mathrm{ng} / \mathrm{mL}$. The otter died a month later, just as euthanasia plans were being made.

Postmortem examination revealed severe, progressive dilated cardiomyopathy and biventricular heart failure as the primary cause of death. Gross necropsy revealed marked dilation of all cardiac chambers, myocardial mottling, reduced thickness of the ventricular walls, extensive pallor of the left papillary muscle and left atrial free wall, and a focally depressed, transparent area of cardiomyocyte loss at the apex (Supplementary Figure S2, available at: avmajournals.avma.org/doi/suppl/10.2460/ ajvr.82.7.529). Systemic indications of congestive heart failure included chronic passive congestion characterized by severe hepatomegaly and hepatic congestion; pleural, pulmonary, pericardial, and peritoneal edema; diffuse venous and lymphatic distension; multifocal venous shunts in the thorax and abdomen; and perimortem circulatory failure and vasodilatory shock characterized by diffusely red oral mucous membranes and mucosal congestion and hemorrhage in the urinary bladder. Histologic evaluation revealed progressive cardiomyocyte swell-
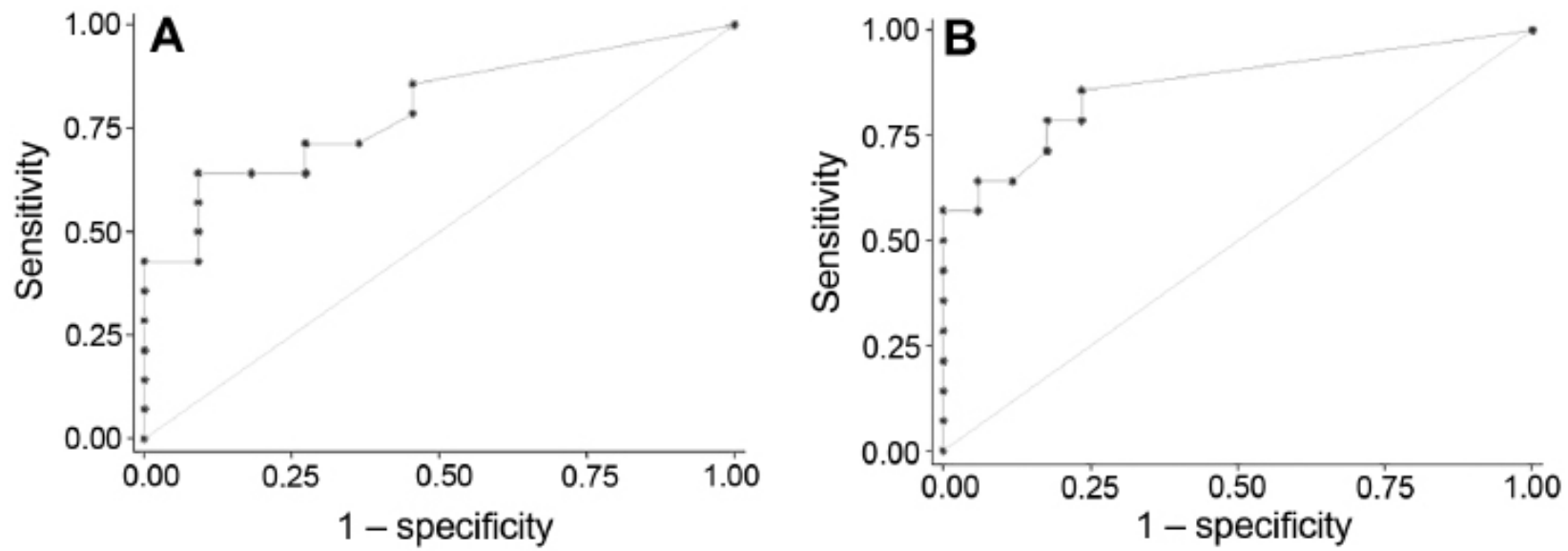

Figure 2-Receiver operating characteristic curves for the use of serum cTnl concentration to detect cardiomyopathy in the free-ranging cases versus free-ranging controls ( $;$; AUC, 0.80$)$ and free-ranging cases versus managed controls (B; AUC, 0.87) of Figure I. True cardiac disease status was determined by gross and histologic examination of cardiac tissue in free-ranging otters and by echocardiography in managed otters. 
Table I-Sensitivity (\%), specificity (\%), and percentage correctly classified as having or not having cardiomyopathy at various cutoff values for serum cTnl concentration in free-ranging southern sea otters (Enhydra lutris nereis) with $(\mathrm{n}=\mathrm{I} 4)$ and without $(\mathrm{I} \mathrm{I})$ cardiomyopathy and healthy managed otters (17).

\begin{tabular}{|c|c|c|c|}
\hline cTnl cutoff (ng/mL) & Sensitivity $(95 \% \mathrm{CI})$ & Specificity $(95 \% \mathrm{CI})$ & Correctly classified (\%) \\
\hline \multicolumn{4}{|c|}{ Free-ranging cases vs free-ranging controls } \\
\hline$\geq 0.007$ & $85.7(57.2-98.2)$ & $54.6(23.4-83.3)$ & 72 \\
\hline$\geq 0.011$ & $78.6(49.2-95.3)$ & $54.6(23.4-83.3)$ & 68 \\
\hline$\geq 0.016$ & $71.4(41.9-91.6)$ & $63.6(30.8-89.1)$ & 68 \\
\hline$\geq 0.019$ & $71.4(41.9-91.6)$ & $72.7(39.0-94.0)$ & 72 \\
\hline$\geq 0.032$ & $64.3(35.1-87.2)$ & $72.7(39.0-94.0)$ & 68 \\
\hline$\geq 0.033$ & $64.3(35.1-87.2)$ & $81.8(48.2-97.7)$ & 72 \\
\hline$\geq 0.037 *$ & $64.3(35.1-87.2)$ & $90.9(58.7-99.8)$ & 76 \\
\hline$\geq 0.042$ & $57.1(28.9-82.3)$ & $90.9(58.7-99.8)$ & 72 \\
\hline \multicolumn{4}{|c|}{ Free-ranging cases vs managed controls } \\
\hline$\geq 0.007$ & $85.7(57.2-98.2)$ & $76.5(50.1-93.2)$ & 81 \\
\hline$\geq 0.008$ & $78.6(49.2-95.3)$ & $76.5(50.1-93.2)$ & 77 \\
\hline$\geq 0.011$ & $78.6(49.2-95.3)$ & $82.4(56.6-96.2)$ & 81 \\
\hline$\geq 0.019$ & $71.4(41.9-91.6)$ & $82.4(56.6-96.2)$ & 77 \\
\hline$\geq 0.027$ & $64.3(35.1-87.2)$ & $88.2(63.6-98.5)$ & 77 \\
\hline$\geq 0.037^{*}$ & $64.3(35.1-87.2)$ & $94.1(71.3-99.9)$ & 81 \\
\hline$\geq 0.038$ & $57.1(28.9-82.3)$ & $94.1(71.3-99.9)$ & 77 \\
\hline$\geq 0.042$ & $57.1(28.9-82.3)$ & $100(80.5-100)$ & 81 \\
\hline
\end{tabular}

*This cutoff value maximized sensitivity and specificity.

ing and depletion, stromal collapse, and interstitial fibrosis but no protozoa. The observed brain lesions were consistent with severe subacute and chronic DA toxicosis. ${ }^{7}$ At the time of death, the otter's serum IgG titer against $T$ gondii was 1:10,240, but disseminated protozoal infection was not histologically observed. Anti-protozoal antibody titers were negative or low throughout the period of managed care (Supplementary Figure S1), suggesting perimortem parasite recrudescence in an immune-compromised animal.

\section{Clinical case study 2: cardiomyopathy associated with a protozoal infection}

A 10-year-old female southern sea otter that was a permanent resident at the Monterey Bay Aquarium had a history of chronic bilateral hip joint degenerative osteoarthritis and was examined while anesthetized every few months. Findings of thoracic auscultation, radiography (VHS, 8.8 vertebrae), and ECG were within reference limits. The initial serum cTnI concentration was $0.058 \mathrm{ng} / \mathrm{mL}$ in September 2017 but decreased when retested 5 and 7 months later (Supplementary Table S2, available at: avmajournals.avma.org/doi/suppl/10.2460/ ajvr.82.7.529). In July 2018, echocardiography performed by a board-certified veterinary cardiologist revealed mild to moderate thickening of the mitral and tricuspid valves, and the serum cTnI concentration increased to $0.128 \mathrm{ng} / \mathrm{mL}$. In January 2019, the otter's mobility became impaired and its appetite decreased, and serum cTnI concentration peaked at $1.165 \mathrm{ng} / \mathrm{mL}$. Serum IgG titers against $S$ neurona were previously negative until this time, when the titer increased 64 fold. One week later, the otter exhibited neurologic signs, including somnolence, head tilt, fine muscle tremors of the head, and blepharospasm. Serum cTnI concentration decreased to $0.123 \mathrm{ng} / \mathrm{mL}$, whereas the serum IgG titer against $S$ neurona increased a further 16-fold. The rapid and substantial increase in anti$S$ neurona antibody was suggestive of acute, rather than recrudescent, infection. In contrast, serum IgG titers against $T$ gondii remained low. Within 2 days, the otter's health status declined precipitously, with abnormal head movements, facial tremors, uncoordinated movements, and grand mal seizures. Heart rate was 90 to 100 beats/min, and ECG revealed a second-degree atrioventricular block (Supplementary Figure S3, available at: avmajournals. avma.org/doi/suppl/10.2460/ajvr.82.7.529) due to high vagal tone from morbidity or sedation. Thoracic radiography revealed a moderate bronchointerstitial pattern and cardiac enlargement (VHS, 9.2 vertebrae). The otter died the next morning.

Necropsy was performed by a veterinary pathologist at The Marine Mammal Center. The pertinent findings included cardiac enlargement with marked focally extensive pallor of the mid to apical left ventricular epicardium and less extensive epicardial pallor extending from the apex to pulmonic trunk on the right ventricle. On cut section, pallor (fibrosis) was present in the subepicardial and subendocardial myocardium of both ventricles and was frequently transmural. There was nodular endocardiosis of both the tricuspid and mitral valves, but lesions were more severe on the right. The lungs had severe diffuse edema and congestion, and the liver was enlarged with a nutmeg pattern of congestion. The brain was edematous with flattened gyri, attenuated sulci, and cerebellar herniation. Histologic findings confirmed the cause of death as diffuse subacute severe myelomeningoencephalitis and multifocal myocarditis associated with an intralesional protozoa identified as $S$ neurona by immunohistochemical staining (Supplementary Figure S4, available at: avmajournals. avma.org/doi/suppl/10.2460/ajvr.82.7.529).5 A more widely disseminated infection included cranial neu- 
ritis, ganglioneuritis, and polyphasic rhabdomyositis with numerous Sarcocystis-like tissue cysts and a lymphoplasmacytic infiltration similar to that in the myocardium. The muscles of respiration, pectoralis muscles, intercostalis muscles, and diaphragm were most severely affected. More chronic cardiac lesions included myxomatous degeneration of the tricuspid and mitral valve leaflets and severe interstitial and subepicardial ventricular myocardial fibrosis. Pulmonary and hepatic congestion were consistent with mitral or tricuspid insufficiency, respectively.

\section{Discussion}

The present study demonstrated the utility of a readily available immunoassay for the detection of cardiomyopathy in southern sea otters. The ROC curves were useful for comparing the overall ability of serum cTnI concentration to discriminate among groups of sea otters with and without cardiomyopathy over the range of possible cutoff values. Some overlap in cTnI concentrations was noted between cases and controls, as has been found in other animals with cardiac disease, ${ }^{20,28,29}$ but future studies with larger sample sizes could help delineate this overlap.

When selecting an optimal cutoff value for serum cTnI concentration to detect cardiomyopathy in sea otters, it is important to consider the usefulness of high sensitivity and specificity in the clinical context of this progressive disease. A test with high sensitivity provides few false-negative results, meaning that fewer cardiomyopathy cases would be missed. Highly sensitive tests are good for disease screening, whereby negative results rule out cardiac disease, particularly when early detection improves prognosis. In human emergency medicine, it is critical to identify acute myocardial infarction because immediate intervention is necessary, indicating that highly sensitivity cTnI assays are crucial. ${ }^{30}$ In contrast, a test with high specificity yields few false-positive results, meaning that fewer sea otters would be misclassified as having cardiomyopathy. Highly specific tests are ideal for disease confirmation, whereby positive results rule in cardiomyopathy, particularly in stranded, debilitated sea otters with multiple systemic conditions that could increase the amount of cTnI in circulation. Cardiac troponin I is not specific for a particular mechanism of myocardial injury and may reflect multiple acute or chronic conditions that damage cardiomyocytes, including congestive heart failure, myocarditis, stress cardiomyopathy, infection, trauma, and other indirect causes such as renal disease and sepsis. ${ }^{15,24,30}$ This underscores the clinical importance of evaluating cardiac and systemic causes of disease and interpreting serum cTnI concentrations in conjunction with physical examination and diagnostic imaging results. ${ }^{19,20}$

Serial measurement of serum cTnI concentration appears to be an important tool for understanding the progression of cardiac disease in sea otters and improving the specificity of cTnI assays ${ }^{30}$ Repeated cTnI testing in humans with acute and chronic cardiac disease has shown that an increase in sequential cTnI values indicates a poor prognosis. ${ }^{31-33}$ Serial cTnI testing in dogs has been used to assess disease progression, response to treatment, and prognosis. ${ }^{19,34}$ The sea otter case studies reported here demonstrated the benefits of serial cTnI testing to improve detection of cardiac disease. In the sea otter with DA-associated cardiomyopathy, the first serum cTnI concentration $\geq$ $0.037 \mathrm{ng} / \mathrm{mL}$ was detected 8.5 months before death, and the peak concentration occurred 4 months before death, whereas the final value decreased markedly but remained above the cutoff value 1 month before death. In the otter with protozoa-associated cardiomyopathy, the first elevated serum cTnI value was identified 1.5 years before death, possibly associated with the insult that resulted in extensive ventricular fibrosis, although subsequently measured concentrations were substantially lower. Serum cTnI concentration increased again 6 months before death, and the highest concentration was detected 10 days before death, whereas the final measurement decreased but remained elevated 4 days before death. The second increase was likely associated with systemic sarcocystosis. In both otters, the peak serum cTnI concentration was detected several days to months before death. The fluctuating cTnI values over time highlighted the importance of serial testing for sea otters in managed care settings where repeated anesthesia, examination, sample collection, and diagnostic tests are a viable option.

False-negative results of the cTnI immunoassay could be common in sea otters with cardiac disease. Cardiomyopathy is progressive and often chronic, whereas CTnI has a short half-life in serum, indicating that only patients with ongoing myocardial injury can be expected to have a high serum cTnI concentration. ${ }^{35}$ In people, serum cTnI concentration increases within 4 to 9 hours after acute myocardial infarction, peaking at 12 to 24 hours and remaining elevated for up to 14 days. ${ }^{36}$ The half-life of cTnI in dogs has also been reported to be hours. ${ }^{15}$ This poses challenges for biomarker detection in free-ranging sea otters with an unknown disease timeline and 1-time opportunistic sample collection. Five of the 14 sea otters with cardiomyopathy had a low serum c'TnI concentration despite moderate to severe cardiac disease at necropsy. Most samples from free-ranging otters were obtained on the same day or 1 day prior to death. In some otters, myocardial injury likely occurred weeks to months before sample collection, with no active cardiomyocyte damage to release c'TnI into circulation. Lasting chronic myocardial injury could still have been present and clinically relevant, yet the short-lived increase in serum cTnI concentration was not captured at the time of sample collection.

Two distinct control groups were used in the present study because both cohorts allowed useful comparisons with otters with cardiomyopathy, yet each control group had limitations. Free-ranging con- 
trols originated from the same wild population as the cases and, therefore, could be expected to have been similarly exposed to factors in their natural environment such as diet, physiologic stressors, and infectious diseases. Another advantage of the free-ranging control group was the definitive absence of heart disease on necropsy. These otters were debilitated and died of various causes, including trauma, which can result in small increases in serum cTnI concentration. In situations where serum samples were obtained near the time of death, agonal changes could have resulted in cardiomyocyte leakage or lysis and subsequent elevation in the amount of cTnI in circulation. In contrast, managed controls were healthy as indicated by results of clinical assessment. However, without postmortem confirmation, they might have had subclinical heart disease or other comorbidities that could have caused an increase in cTnI release. Exhibit animals also represent a cohort different from free-ranging otters, and comparisons between the 2 may be obscured by differences in life history characteristics, diet, and pathogen or toxin exposure. Different comparison groups and cutoff values for serum cTnI concentration should be considered, depending on the sea otter population and consequences of a given test result for decisions regarding management, treatment, and prognosis.

In zoo and wildlife medicine, a lack of reference data and validated tests has impeded the identification and treatment of cardiac disease. A study ${ }^{37}$ of the use of a point-of-care analyzer to measure serum or plasma cTnI concentration in 36 species at the Zoological Society of London shows that cTnI may be a promising biomarker of cardiac disease in many nondomestic mammals. Stranded cetaceans commonly exhibit capture myopathy syndrome, which is characterized by acute cardiac injury. ${ }^{38}$ Plasma cTnI concentrations have been measured in live-stranded cetaceans and captive bottlenose dolphins (Tursiops truncatus), indicating high values in stranded animals, some of which had acute myocardial necrosis..$^{39}$

Regardless of etiology, myocardial damage and a high serum cTnI concentration have been associated with an increased risk of death in the short and long term in various species, emphasizing the utility of serum cTnI concentration as a prognostic indicator. ${ }^{16,19,33,40-42}$ This clinical tool may be particularly useful in wildlife rehabilitation. Harbor seal (Pboca vitulina) pups that die during or after rehabilitation have been found to have a significantly higher antemortem serum cTnI concentration at admission than do those that survive after release. ${ }^{42}$ Although this was not the aim of the present study, we observed that healthy managed sea otters with the highest cTnI values were those with unsuccessful releases into the wild. One died of emaciation 2 weeks after release. The other was recaptured 3 weeks after release, received medical treatment for shark bite wounds and acanthocephalan peritonitis, and was subsequently managed under human care.
The association between DA exposure and cardiomyopathy has been well described in southern sea otters ${ }^{1-3,7}$ and California sea lions. ${ }^{8,9}$ Postmortem serum cTnI testing in sea lions with DA-associated cardiomyopathy revealed no significant difference in median cTnI concentration among animals with acute DA toxicosis and chronic DA toxicosis and controls with no cardiac abnormalities, although several sea lions with acute and chronic toxicosis had a markedly high serum cTnI concentration. ${ }^{9}$ Both acute and ongoing myocardial damage can lead to measurable increases in circulating troponin concentrations, indicating that a high cTnI concentration alone cannot distinguish between acute and chronic cardiomyopathy. In addition, the small number of controls in the sea lion study likely limited the ability to detect a significant difference in serum cTnI concentration.

In the present study, the first clinical case example illustrated the combined effects of DA on the heart and brain, with cardiac abnormalities that temporally matched the observed subacute and chronic DA-associated brain lesions. Previous DA exposure could not be accurately assessed with biochemical testing given the chronicity of the DA-associated lesions. However, our previous research demonstrated that sea otters are at risk of fatal cardiomyopathy if exposed to high DA levels in the past year. ${ }^{3}$ The largest recorded DA event in California occurred in the spring of 2015 after anomalously warm ocean conditions initiated an extensive and prolonged toxic algal bloom. ${ }^{43}$ Case study 1 became stranded in May 2016 in Monterey Bay, a region severely impacted in the 2015 DA event. ${ }^{43}$ The year interval between toxic bloom and stranding provides additional evidence that DA exposure likely played a role in cardiac disease development.

An important limitation of the study reported here was that the immunoassay used to measure serum cTnI concentration has not been validated for sea otters, although cTnI expression is widely conserved across mammals. ${ }^{15-18}$ Furthermore, the measured cTnI values were specific to this assay and correlation with point-of-care tests could be an area of future research. Additional studies could explore other serologic biomarkers of cardiac disease, such as $\mathrm{N}$ terminal proatrial and probrain natriuretic peptides, which have been shown to increase with congestive heart failure severity, facilitate diagnosis, and guide treatment. ${ }^{44-46}$ These biomarkers are highly speciesspecific; sea otter sequences have not been determined and may not be homologous with canine and feline peptide sequences, limiting cross-reactivity and adequate test performance. Development and validation of a sea otter-specific natriuretic peptide assay may yield another useful tool in cardiomyopathy diagnosis.

Findings of the present study may be useful to inform triage decisions, influence case management, and optimize treatment protocols to benefit managed sea otters in zoos, aquariums, and rehabili- 
tation centers and free-ranging sea otters. The US Fish and Wildlife Service has determined that oil spills, DA, and climate change are among the key threats to recovery of this endangered species. ${ }^{47} \mathrm{Re}$ liable information on the impacts of DA exposure and cardiomyopathy is essential to guide recovery efforts and effective oil-spill response. We believe that measurement of serum cTnI concentration, when combined with other clinical tools, can greatly improve our ability to diagnose cardiac disease in sea otters. Serial cTnI testing will be particularly useful for long-term management of chronic cardiac conditions in resident exhibit sea otters. Measurement of serum cTnI concentration may also provide prognostic information for injured, oil-exposed, or diseased free-ranging sea otters related to the likelihood of successful rehabilitation and release.

\section{Acknowledgments}

Funded by the California Department of Fish and Wildlife's Oil Spill Prevention and Administration Fund through the Oiled Wildlife Care Network at the Karen C. Drayer Wildlife Health Center, School of Veterinary Medicine, University of California-Davis.

The authors declare that there were no conflicts of interest.

The authors thank Francesca Batac, Erin Dodd, Barbie Halaska, Jaclyn Isbell, and Carlos Rios for providing the laboratory samples and data; Shawn Johnson, Emily Whitmer, Abby McClain, Brittany Stevens, and Christine Parker-Graham for providing veterinary care and expertise, with assistance from Marissa Young, Erin Lenihan, Jessica Folck, Lauren Campbell, Sophie Guarasci, Sarah Pattison, and Juli Barron; Chris DeAngelo and Karl Mayer for providing animal care; and Robynne Gomez and Phillip Guadiano for performing the cTnI assays.

\section{Footnotes}

a. Advia Centaur CP ultra-TnI immunoassay, Siemens Healthcare Diagnostics Inc, Tarrytown, NY.

b. R: A language and environment for statistical computing, ver sion 3.5.0, R Foundation for Statistical Computing, Vienna, Austria.

c. Stata, version 14.2, StataCorp, College Station, Tex.

\section{References}

1. Miller MA, Moriarty ME, Henkel LA, et al. Predators, disease, and environmental change in the nearshore ecosystem: mortality in southern sea otters (Enbydra lutris nereis) from 1998-2012. Front Mar Sci 2020;7:582.

2. Kreuder C, Miller MA, Lowenstine LJ, et al. Evaluation of cardiac lesions and risk factors associated with myocarditis and dilated cardiomyopathy in southern sea otters (Enbydra lutris nereis). Am J Vet Res 2005;66:289-299.

3. Moriarty ME, Tinker MT, Miller MA, et al. Exposure to domoic acid is an ecological driver of cardiac disease in southern sea otters. Harmful Algae 2021;101:101973.

4. Kreuder C, Miller MA, Jessup DA, et al. Patterns of mortality in southern sea otters (Enhydra lutris nereis) from 19982001. J Wildl Dis 2003;39:495-509.

5. Miller MA, Conrad PA, Harris M, et al. A protozoal-associated epizootic impacting marine wildlife: mass-mortality of southern sea otters (Enhydra lutris nereis) due to Sarcocystis neurona infection. Vet Parasitol 2010;172:183-194.

6. Scholin CA, Gulland F, Doucette GJ, et al. Mortality of sea lions along the central California coast linked to a toxic diatom bloom. Nature 2000;403:80-84.

7. Miller MA, Moriarty ME, Duignan PJ, et al. Clinical signs and pathology associated with domoic acid toxicosis in southern sea otters (Enbydra lutris nereis). Front Mar Sci 2021;8:208.

8. Silvagni PA, Lowenstine LJ, Spraker T, et al. Pathology of domoic acid toxicity in California sea lions (Zalopbus californianus). Vet Patbol 2005;42:184-191.

9. Zabka TS, Goldstein T, Cross C, et al. Characterization of a degenerative cardiomyopathy associated with domoic acid toxicity in California sea lions (Zalopbus californianus). Vet Patbol 2009;46:105-119.

10. Teitelbaum JS, Zatorre RJ, Carpenter S, et al. Neurologic sequelae of domoic acid intoxication due to the ingestion of contaminated mussels. N Engl J Med 1990;322:1781-1787.

11. Perl TM, Bédard L, Kosatsky T, et al. An outbreak of toxic encephalopathy caused by eating mussels contaminated with domoic acid. $N$ Engl J Med 1990;322:1775-1780.

12. Jing J, Petroff R, Shum S, et al. Toxicokinetics and physiologically based pharmacokinetic modeling of the shellfish toxin domoic acid in nonhuman primates. Drug Metab Dispos 2018;46:155-165.

13. Lefebvre KA, Frame ER, Gulland F, et al. A novel antibodybased biomarker for chronic algal toxin exposure and subacute neurotoxicity. PLoS One 2012;7:e36213.

14. Sharma S, Jackson PG, Makan J. Cardiac troponins. J Clin Patbol 2004;57:1025-1026.

15. Langhorn R, Willesen JL. Cardiac troponins in dogs and cats $J$ Vet Intern Med 2016;30:36-50.

16. Oyama MA, Sisson DD. Cardiac troponin-I concentration in dogs with cardiac disease. J Vet Intern Med 2004;18:831839.

17. Serra M, Papakonstantinou S, Adamcova M, et al. Veterinary and toxicological applications for the detection of cardiac injury using cardiac troponin. Vet J 2010;185:50-57.

18. O'Brien PJ, Smith DE, Knechtel TJ, et al. Cardiac troponin I is a sensitive, specific biomarker of cardiac injury in laboratory animals. Lab Anim 2006;40:153-171.

19. Fonfara S, Loureiro J, Swift S, et al. Cardiac troponin I as a marker for severity and prognosis of cardiac disease in dogs. Vet J 2010;184:334-339.

20. Langhorn R, Willesen JL, Tarnow I, et al. Evaluation of a highsensitivity assay for measurement of canine and feline serum cardiac troponin I. Vet Clin Patbol 2013;42:490-498.

21. Newby LK, Jesse RL, Babb JD, et al. ACCF 2012 expert consensus document on practical clinical considerations in the interpretation of troponin elevations: a report of the American College of Cardiology Foundation Task Force on Clinical Expert Consensus Documents. J Am Coll Cardiol 2012;60:2427-2463.

22. Gunther-Harrington CT, Moriarty ME, Field CL, et al. Transthoracic echocardiographic evaluation and serum cardiac troponin values in anesthetized healthy female southern sea otters (Enbydra lutris nereis). J Zoo Wildl Med 2021;52:in press.

23. Frame ER, Lefebvre KA. ELISA methods for domoic acid quantification in multiple marine mammal species and sample matrices. NOAA Technical Memorandum NMFS-NWFSC-122. Seattle: Northwest Fisheries Science Center, National Marine Fisheries Service, National Oceanic and Atmospheric Administration, US Department of Commerce, 2013.

24. Porciello F, Rishniw M, Herndon WE, et al. Cardiac troponin $I$ is elevated in dogs and cats with azotaemia renal failure and in dogs with non-cardiac systemic disease. Aust Vet J 2008;86:390-394.

25. Saenger AK, Jaffe AS, Body R, et al. Cardiac troponin and natriuretic peptide analytical interferences from hemolysis and biotin: educational aids from the IFCC Committee on Cardiac Biomarkers (IFCC C-CB). Clin Chem Lab Med 2019;57:633640.

26. Greiner M, Pfeiffer D, Smith RD. Principles and practical application of the receiver-operating characteristic analysis for diagnostic tests. Prev Vet Med 2000;45:23-41.

27. Stepien RL, Benson KG, Forrest LJ. Radiographic measurement of cardiac size in normal ferrets. Vet Radiol Ultrasound 1999;40:606-610.

28. Spratt DP, Mellanby RJ, Drury N, et al. Cardiac troponin I: evaluation of a biomarker for the diagnosis of heart disease in the dog. I Small Anim Pract 2005;46:139-145.

29. Oyama MA, Solter PF. Validation of an immunoassay for 
measurement of canine cardiac troponin-I. J Vet Cardiol 2004;6:17-24.

30. de Lemos JA. Increasingly sensitive assays for cardiac troponins: a review. JAMA 2013;309:2262-2269.

31. Kawahara C, Tsutamoto T, Sakai H, et al. Prognostic value of serial measurements of highly sensitive cardiac troponin I in stable outpatients with nonischemic chronic heart failure. Am Heart J 2011;162:639-645.

32. Keller T, Zeller T, Ojeda F, et al. Serial changes in highly sen sitive troponin I assay and early diagnosis of myocardial infarction. JAMA 2011;306:2684-2693.

33. Stanton EB, Hansen MS, Sole MJ, et al. Cardiac troponin I, a possible predictor of survival in patients with stable congestive heart failure. Can J Cardiol 2005;21:39-43.

34. Polizopoulou ZS, Koutinas CK, Dasopoulou A, et al. Serial analysis of serum cardiac troponin I changes and correlation with clinical findings in 46 dogs with mitral valve disease. Vet Clin Patbol 2014;43:218-225.

35. Dunn ME, Coluccio D, Hirkaler G, et al. The complete pharmacokinetic profile of serum cardiac troponin $I$ in the rat and the dog. Toxicol Sci 2011;123:368-373

36. Bertinchant J-P, Larue C, Pernel I, et al. Release kinetics of serum cardiac troponin I in ischemic myocardial injury. Clin Biochem 1996;29:587-594.

37. Feltrer Y, Strike T, Routh A, et al. Point-of-care cardiac troponin $\mathrm{I}$ in non-domestic species: a feasibility study. $J$ Zoo Aquar Res 2016;4:99-103.

38. Herráez P, Espinosa de los Monteros A, Fernández A, et al. Capture myopathy in live-stranded cetaceans. Vet $J$ 2013;196:181-188

39. Câmara N, Sierra E, Fernández A, et al. Increased plasma cardiac troponin I in live-stranded cetaceans: correlation with pathological findings of acute cardiac injury. Sci Rep 2020;10:1555

40. Antman EM, Tanasijevic MJ, Thompson B, et al. Cardiacspecific troponin I levels to predict the risk of mortality in patients with acute coronary syndromes. $N$ Engl J Med 1996;335:1342-1349.

41. Eggers KM, Lagerqvist B, Venge P, et al. Persistent cardiac troponin I elevation in stabilized patients after an episode of acute coronary syndrome predicts long-term mortality. Circulation 2007;116:1907-1914.

42. Fonfara S, Sundermeyer J, Casamian Sorrosal D, et al. Usefulness of serum cardiac troponin I concentration as a marker of survival of harbor seal (Pboca vitulina) pups during rehabilitation. J Am Vet Med Assoc 2016;249:1428-1435.

43. McCabe RM, Hickey BM, Kudela RM, et al. An unprecedented coastwide toxic algal bloom linked to anomalous ocean conditions. Geophys Res Lett 2016;43:10366-10376.

44. Palazzuoli A, Gallotta M, Quatrini I, et al. Natriuretic peptides (BNP and NT-proBNP): measurement and relevance in heart failure. Vasc Health Risk Manag 2010;6:411-418.

45. Connolly DJ, Soares Magalhaes RJ, Syme HM, et al. Circulating natriuretic peptides in cats with heart disease. $J$ Vet Intern Med 2008;22:96-105.

46. Oyama MA, Sisson DD, Solter PF. Prospective screening for occult cardiomyopathy in dogs by measurement of plasma atrial natriuretic peptide, B-type natriuretic peptide, and cardiac troponin-I concentrations. Am J Vet Res 2007;68:42-47.

47. US Fish and Wildlife Service. Southern sea otter (Enhydra lutris nereis) 5-year review: summary and evaluation. Ventura, Calif: US Fish and Wildlife Service, 2015. 